\title{
Preparation of a self-curable unsaturated polyester with vinyl double bonds and Investigation of its self-curing properties
}

\author{
Haiyan Li, Fangfang Zhu, and Chongwen Li \\ Institute of Materials Science and Engineering, Ocean University of China, Songling Road, Number238, Qingdao 266100, China
}

\begin{abstract}
In this paper, a kind of self-curable unsaturated polyester PGA-UP with vinyl groups on the end of molecule chains was synthesized through esterification reaction of glycidol with acrylic acid. The self-curable function of unsaturated polyester was put forward as a new solution to air pollution problems caused by the crosslinking monomer's volatilization in unsaturated polyesters (UP) industry. The synthetic method, reaction process, molecular structure and self-curing properties were studied. Investigation demonstrates that the unsaturated polyester resin PGA-UP could be synthesized through a two-step process and the liquid product was a mixed outcome of different extent esterification. The unsaturated polyester resin could be self-cured through the free radical reaction of vinyl groups on the end of the molecules when they were initiated by $1 \%$ benzoyl peroxide (BPO) at $80^{\circ} \mathrm{C}$ for 1 hours and $120^{\circ} \mathrm{C}$ for 2 hours with no cross-linking monomers. The intension strength of the self-cured PGA-UP is $13.5 \mathrm{Mpa}$, close to the $15.6 \mathrm{MPa}$ of an usual unsaturated polyester S-UP made in our lab. The self-curable PGA-UP presents good potential application and the research will contribute to improve the environmental problems caused by volatile of cross-linking monomers in unsaturated polyester applications. 1H-NMR and FT-IR were used to characterize.
\end{abstract}

\section{Introduction}

The thermosetting unsaturated polyester (UP) is one of the most widely used resin in modern plastic industry. The double bonds on the main chain of UP is unable to be cured through free radical homo-polymerization and it is usually dissolved in some vinyl monomer and thermally crosslink through free-radical copolymerization with vinyl monomers initiated by peroxide [1]. However, styrene (St), the commonly used cross-linking monomer, produces toxic volatile during the curing process. In past year, a lot of measures have been taken, and most of them are focused on how to lower the amount of the volatile or to use lower toxic or non-toxic monomers as substitute and all demonstrate kinds of limits. Few of them are focused on the structure and function of unsaturated polyesters [1].

On different view from above, in our lab, it is believed that the unsaturated polyester with new molecule structures and self-curable properties can present a new solution to the air pollution caused by cross-linking monomers. Some new unsaturated polyesters with self-curable function have been designed and prepared, which could self crosslink without other monomers and of course the air pollution problem is avoid [2-3]

At present, self-curable unsaturated polyesters have attracted great attentions in the research field of medical polymer and tissue engineering [2-7]. Among them, the majority are the unsaturated polyesters with nucleophilic pendant groups on side chain, such as hydroxyl, thiol or amino group, which usually was prepared with malefic anhydride (MA) as one of the raw materials $[2,3,6]$. As the double bonds located on main chain present low free radical activity, it is difficult for such UPs to selfcrosslink through the free radical homo-polymerization. Differently, they come into self-crosslinking through the nucleophilic addition reaction of nucleophilic pendant groups with the double bonds [2-3]. However, the selfcurable UP resin is difficult to process because of its high viscosity and the high temperature for curing. Meanwhile, much fault existing in the product after cured and the low curing extent result in restricted applications. At present, kinds of self-curable unsaturated polyesters are designed and synthesized for biomaterials, but it is much insufficient for potential applications. The types and applications of self-curable unsaturated polyesters need great expansion.

In order to solve the air pollution problem and improve the curing process and properties after cured to expand the application of self-curable UPs, one kind of unsaturated polyester (PGA-UP) with vinyl bonds located at the end of molecular main chain was made in our lab, through esterifization of glycidol (GL) with acrylic acid (AC), with the existance of radical inhibitor. The PGAUP obtained presented low viscosity at room temperature and could be thermally self-cured without the crosslinking monomer. Compared with the double bonds in the middle of molecular chain of common unsaturated polyesters, the vinyl bonds on the end of molecular 
chains presented higher molecular mobility, lower steric hindrance and, consequently, present higher selfcrosslinking activity. In the synthesis of the PGA-UP, in order to avoid the radical homo-polymerization of the vinyl double bonds during the the esterifization process, temperature was controlled strictly under $118^{\circ} \mathrm{C}$ which was much lower than the usual ersterifizaion temperature. As a result, the key point during the sythesis is how to promote the esterfization reaction rate under the low temperature. Here benzene and toluene mixture was added as water carrying agent and its mixing ratio was adjusted to control the reflux temperature of the reaction system. The synthetic technological process, reaction mechanism, molecular structure and self-curing properties were studied.

\section{Experimental}

\subsection{Materials}

Acrylic acid (A.R), toluene (A.R), p-hydroxyl phenol (A.R), anhydrous zinc chloride (A.R), xylene (A.R), benzoyl peroxide (BPO) (A.R) and N, N-dimthylben zylamine $(99 \%)$, were obtained from Tianjin Bodi Chemical Reagent Factory (Tianjin City, China) without further treatment. Glycidol (GL, 96\%) was obtained from Sinopharm Chemical Reagent Co., Ltd. (Shanghai City, China) without further treatment.

\subsection{Experimental}

\subsubsection{Synthesis of the self-curable resin PGA-UP}

In this reaction, one molecule Glycidol (GL) is designed to be esterified by three Acrylic acid molecules. In order to make the esterifying reaction complete, a third excessive molar dose of AC was added. The experimental process was shown as follows:

GL22.44g (0.30mol), AC $3.603 \mathrm{~g}(0.05 \mathrm{~mol}), \mathrm{N}, \mathrm{N}-$ dimthylbenzylamine $0.02 \mathrm{~g}(1 \%$ of GL amount), phydroxyl phenol $0.014 \mathrm{~g}$ (HP, $0.05 \%$ of GL amount) and anhydrous zinc chloride $0.108 \mathrm{~g}$ as esterification catalyst were placed into a $250 \mathrm{ml}$, four-necked, round-bottomed flask equipped with an agitator, a thermometer, a $\mathrm{N}_{2}$ tube, a reflux condenser pipe and a constant pressure funnel. The system was run and slowly heated and kept at 74$80{ }^{\circ} \mathrm{C}$. AC $28.824 \mathrm{~g}(0.40 \mathrm{~mol})$ in the constant pressure funnel was dropped into the reaction system at a constant speed during 5 hours. The reaction extent was monitored by the change of viscosity and the acid value of the system.

When the acid value in reactants solution could not decrease further, the reflux condenser pipe was displaced by water segregator with full of water carrying agent, the mixture of benzene and toluene (volume ratio being 3:1). The reaction system was heated slowly and the water carrying agent was also added into the system to keep the reflux temperature of the system band to $110-112^{\circ} \mathrm{C}$. The residual AC $43.236 \mathrm{~g}(0.60 \mathrm{~mol})$ was added into the constant pressure funnel and slowly dropped into the system for about 5 hours. During the reaction process, the system kept reflux to drive away the byproduct water into the water segregator for enhancing the esterification conversion further. With the reaction going further, the reflux temperature increased slowly. In order to control the reflux temperature varied between $110-118^{\circ} \mathrm{C}$, the water carrying agent was added occasionally as dependent. The acid value was monitored every hour. When the acid value did not decrease further, the water segregator was displaced by a negative pressuredistillation system with water-ring vacuum pump. The excessive $\mathrm{AC}$ was distilled slowly under $68-72^{\circ} \mathrm{C}$ for about 1.5 hours until no distillate out. The reaction product was extracted to remove the solid byproduct and distilled with oil-ring vacuum pump. A distillate of $58^{\circ} \mathrm{C}$ was collected as our product, PGA-UP, a transparent light brown liquid at room temperature. ${ }^{1} \mathrm{H}-\mathrm{NMR}$ and FT-IR were used to characterize the product. Both the acid value and hydroxyl value of the product were tested.

\subsubsection{Curing experiments of PGA-UP resin}

One PGA-UP sample mixed with the free radical initiator BPO being $1.0 \%$ of the PGA-UP amount, was named as PGA-UP-1. After the dissolution of BPO, The mixture was mounted into 7 molds respectively and heated respectively at different constant temperature as $60^{\circ} \mathrm{C}$, $80^{\circ} \mathrm{C}, 90^{\circ} \mathrm{C}, 100^{\circ} \mathrm{C}, 120^{\circ} \mathrm{C}, 140^{\circ} \mathrm{C}, 150^{\circ} \mathrm{C}$ for enough time to self crosslink. Another PGA-UP sample with no BPO addition was named as PGA-UP-2 and was carried on the same experiments as PGA-UP-1. Some of the Samples before and after cured were characterized by FT-IR spectra.

\subsection{Analysis and Characterization}

${ }^{1}$ H-NMR spectra were carried out on a JEOL JNM-ECP 600 NMR spectrometer (Japan Electronics Co., Ltd.), with the resonance frequency of $600 \mathrm{MHz}$. Infrared spectra were performed on a Bruker TENSOR27 Fourier transform spectrometer (Bruker Instruments Inc., Germany) at room temperature, measuring range 400 4 $000 \mathrm{~cm}^{-1}$.

The intension strength were measured on an Instron 1122 testing instrument with $80 \mathrm{~mm} \times 10 \mathrm{~mm} \times 3 \mathrm{~mm}$ specimens in accordance with GB/T2569-1995 at a drawing rate of $5 \mathrm{~mm} / \mathrm{min}$. The samples for intension strength test were made as follows: The PGA-UP liquid mixed with BPO being $1.0 \%$ of the PGA-UP amount, was mounted into the molds, and was thermally cured at $80^{\circ} \mathrm{C}$ for one hour and then $120^{\circ} \mathrm{C}$ for 2 hours. After the curing process, the samples were polished and their dimensions were measured for the intension strength test next.

\section{Results and discussions}

\subsection{Preparation of the PGA-UP resin}


During the esterification of the GL and $\mathrm{AC}$, If $\mathrm{AC}$ was added fast or the reaction temperature was high above $80^{\circ} \mathrm{C}$, the reaction heat could not dissipate well, local overheating would lead to gelatin and the synthesis failed. In order to make the esterificaiton carry on smoothly without gelatin, free radical inhibitor was necessary, the reaction temperature must be strictly controlled below $80^{\circ} \mathrm{C}$, AC must be dropped slowly into the reaction system and the system should be stirred strongly to dissipate the reaction heat. During the reaction step at 74$80^{\circ} \mathrm{C}$, the viscosity of the system increased gradually and at last it increased no more. No water product was observed.

When the reaction was not able to go further below $80^{\circ} \mathrm{C}$, the reaction temperature was heated slowly to $110^{\circ} \mathrm{C}$. With the existence of the water carrying agent, the system reflux at $110-112^{\circ} \mathrm{C}$ and water byproduct began to be distilled into the water segregator. The viscosity of the system began to increase gradually again. In the second step reaction, with the reaction's going on, the reflux temperature will slowly increase. In order to control the reflux temperature, appropriate dose water carrying reagent was added occasionally to control the temperature varied between $110-118^{\circ} \mathrm{C}$. If $\mathrm{AC}$ was added faster or the reflux rate was fast or the reaction temperature was higher than $118^{\circ} \mathrm{C}$, gelatin may occur and solid grain byproduct would come into being. Therefore, it is very important to control the reaction temperature and the reaction rate.

Based on the reaction behavior, we assume that the reaction carries on by two steps as shown in Figure 1. Firstly, GL reacted with AC through the ring opening reaction for forming ester group at $80^{\circ} \mathrm{C}$; secondly, the intermediate products with two hydroxyl groups further reacted with two ACs to form another two ester groups at $110^{\circ} \mathrm{C}-118^{\circ} \mathrm{C}$. As shown in Figure 1, one GL molecule needs to react with three $\mathrm{AC}$ molecules to produce the unsaturated polyester pre-polymer with three vinyl bonds on the end of molecule chain, named as PGA-UP. In order to make the hydroxyl produced in the reaction process being esterifed completely, the molar ratio of GL and $\mathrm{AC}$ is 1:4 with $\mathrm{AC}$ excessive.
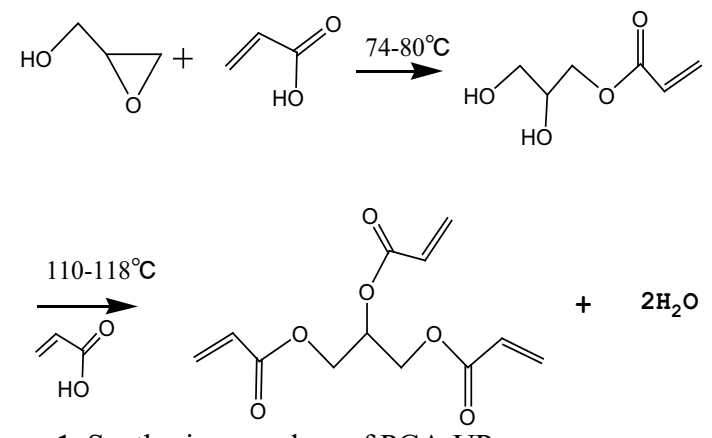

Figure 1. Synthesis procedure of PGA-UP.

The acid value of the liquid product obtained is 57.8 $\mathrm{mg} \mathrm{KOH} / \mathrm{g}$ and the hydroxyl value is $122.5 \mathrm{mg} \mathrm{KOH} / \mathrm{g}$. Based on the hydroxyl value and acid value, the reaction conversion extent of hydroxyl group is about $80.7 \%$, the conversion rate of the carbonyl group is about $81.5 \%$.
The above result indicates that the hydroxyl of GL has not been esterified completely. We assumed that the yellow liquid product was a mixture of compounds produced by GL with different esterifying reaction degree with $\mathrm{AC}$, which is verified by the 1H-NMR and FT-IR spectra. The molecule structures are listed in Figure 2. The 1H-NMR with acetone-d6 as a solvent and FT-IR are shown in Figure 3 and Figure 4.

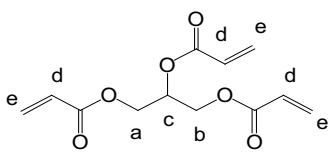

product 1



product 3

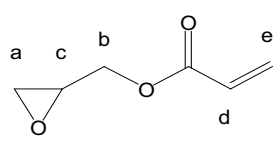

product 4

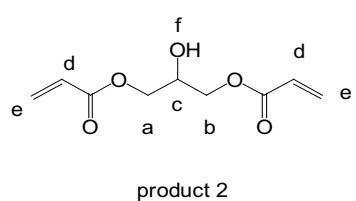

Figure 2. Molecule structure of the liquid product.

As shown in Figure 2, product1 is the target product PGA-UP as desired which is synthesized through the esterifying reaction of one molecule GL with three molecules of AC. As shown in the 1H-NMR spectrum, the peaks at $6.35 \mathrm{ppm}$ and $5.89 \mathrm{ppm}$ are designated for the protons e, from the vinyl double bonds on the end of the molecular chain, and the peak at $6.10 \mathrm{ppm}$ is designated for the proton of the vinyl double bonds named as $\mathrm{d}$. The peaks at $4.39 \mathrm{ppm}$ is designated for the protons $\mathrm{a}$ and $\mathrm{b}$, and the peaks at $5.21 \mathrm{ppm}$ is designated for the protons $\mathrm{c}$.

Product 2 is produced through the esterifying reaction of one molecule GL with two molecules of AC, with a residual hydroxyl group unreacted on the molecule structure. The peaks at 4.24-4.21 ppm are designated for the protons of $a$ and $b$ in Product 2. The peaks produced by the proton $c$ of product 2 are the triplet peaks at $4.39 \mathrm{ppm}$ which are overlapped by the peaks of the protons $a$ and $b$ of product 1 . The peaks produced by the protons $\mathrm{d}$ and $e$ of product 2 are at the same location with those $\mathrm{d}$ and $e$ of product 1 .

Product 3 and 4 are produced through the esterifying reaction of one molecule GL with one molecule AC. Product 3 is produced by the ring-opening reaction of GL with AC. As shown in Figure 3, the peaks for the protons $a$ are at $3.56-3.59 \mathrm{ppm}$, the peaks for the protons $b$ are at 4.16-4.12ppm, and the multiplet peaks for the protons $c$ are at $3.9 \mathrm{ppm}$. The peaks produced by the protons $d$ and $\mathrm{e}$ of the vinyl double bonds of product 3 are at the same location with the protons $d$ and $e$ of product 1 and product 2 . The peak at $4.72 \mathrm{ppm}$ are designated for the proton $f$ of hydroxyl groups unreacted in product 2 and 3.Product 4 is produced by the esterifying reaction of the hydroxyl group on GL with the carboxyl group on AC. The protons $a$ and $c$ are located on the epoxy ring. The peaks for the two protons $a$ are at $2.47-2.52 \mathrm{ppm}$ and $2.75 \mathrm{ppm}$ respectively, and the peaks for the protons $c$ are at $3.30 \mathrm{ppm}$. The peaks for $b$ are at the same location with the proton $b$ of product 3 . The peaks produced by the protons $d$ and $e$ are at the same location with those of product 1 . Based on the peak intensity, we can estimate 
that the liquid product is mainly composed by product 1 , 2 and 3 , and the amount of product 4 is much less than the other three.

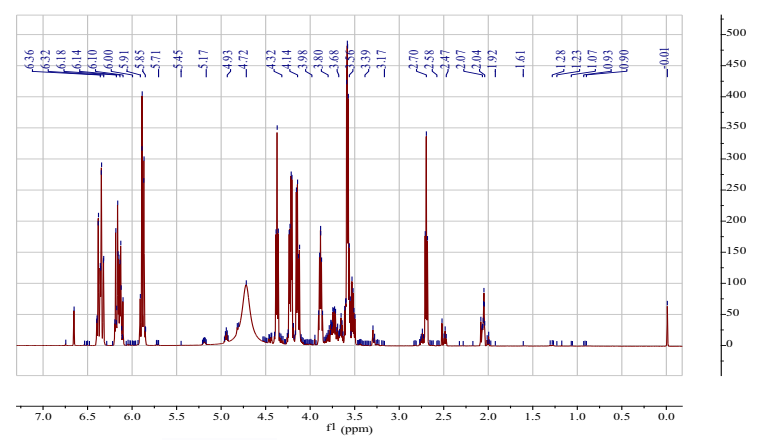

Figure 3. The ${ }^{1} \mathrm{H}-\mathrm{NMR}$ spectra of PGA-UP.

As shown in the FT-IR spectrum in Figure 4, the peaks at $1635 \mathrm{~cm}^{-1}$ and $1618 \mathrm{~cm}^{-1}$ are due to stretching vibration of $\mathrm{C}=\mathrm{C}$ double bonds; the small peak at $3034 \mathrm{~cm}^{-1}$ also shows the existence of $\mathrm{C}=\mathrm{C}$ double bonds; the absorption at $1410 \mathrm{~cm}^{-1}$ is caused by in-plane bending vibration of the $\mathrm{C}-\mathrm{H}$ of vinyl double bonds; the absorption at $985 \mathrm{~cm}^{-1}$ and $810 \mathrm{~cm}^{-1}$ are caused by outplane bending vibration of the vinyl double bonds on the chain end. All the above absorptions indicate the existence of the vinyl double bonds. The peak at $1722 \mathrm{~cm}^{-}$ ${ }^{1}$ is for the $\mathrm{C}=\mathrm{O}$ stretching vibration; Peaks at $1165 \mathrm{~cm}^{-1}$ and $1215 \mathrm{~cm}^{-1}$ are caused by stretching vibration of $\mathrm{C}-\mathrm{O}$. The absorption at $3335 \mathrm{~cm}^{-1}$ is caused by stretching vibration of residual hydroxyl group unreacted of product 2 and 3 ; the small absorption at $915 \mathrm{~cm}^{-1}$ is attributed to epoxide group from product 4 . The result is consistent with what shown in the ${ }^{1} \mathrm{H}-\mathrm{NMR}$ spectrum.

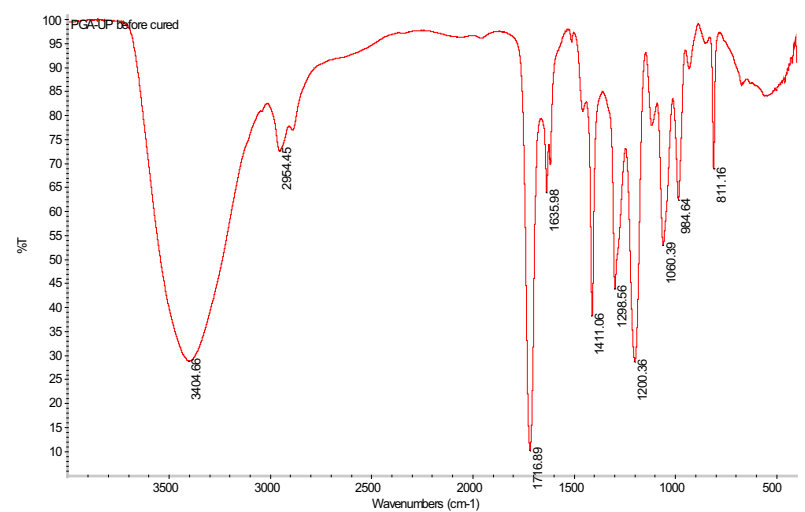

Figure 4. FT-IR spectra of PGA-UP before cured.

\subsection{The self-curable properties of PGA-UP}

After the curing experiments at different heating temperature, we found that the samples of PGA-UP-1, containing $1 \% \mathrm{BPO}$ initiator, could be thermally self-cured into a light yellow transparent solid after heated at $80^{\circ} \mathrm{C}$ for 1 hour and became more hard after heated at $120^{\circ} \mathrm{C}$ for 2 hours .However, the samples PGA-UP-2 without $\mathrm{BPO}$ as initiator could be slowly cured until heated at $150^{\circ} \mathrm{C}$ and completely cured until heated at $180^{\circ} \mathrm{C}$ for 2 hours. Obviously, the existence of the radical initiator
BPO greatly induced the self cross-linking reaction rate of PGA-UP.

As shown in the FT-IR spectrum of PGA-UP after cured in Figure 5, the peaks due to the vinyl double bonds at $1635 \mathrm{~cm}^{-1}, 1618 \mathrm{~cm}^{-1}, 3037 \mathrm{~cm}^{-1}, 985 \mathrm{~cm}^{-1}$ and $810 \mathrm{~cm}^{-1}$ all disappeared and other peaks changed little compared with those before cured in Figure 4. Based on the above experiments, we assumed that the double bonds of PGAUP can self crosslink through radical homopolymerization due to the higher radical polymerization reactivity when thermally initiated by $\mathrm{BPO}$ at $80^{\circ} \mathrm{C}$. PGA-UP can be self cured without other monomers. The self curing reaction schematic diagram is shown as Figure 6.

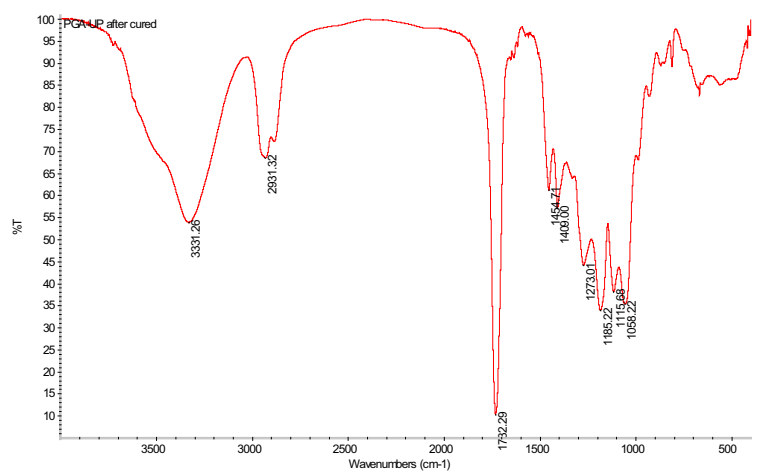

Figure 5. FT-IR spectra of PGA-UP after cured.

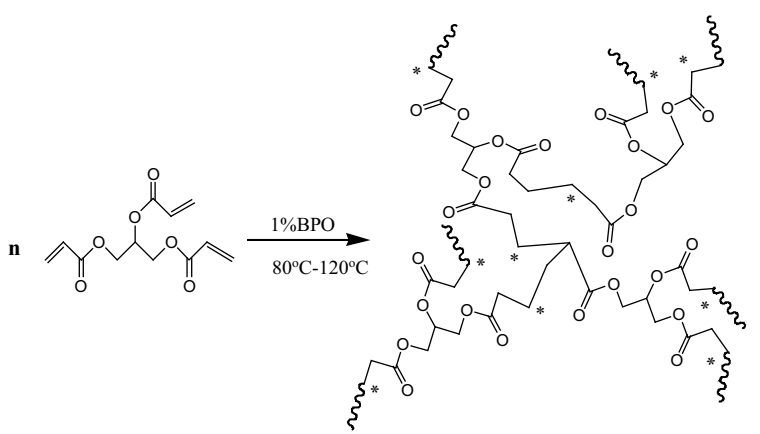

Figure 6. The curing process scheme of PGA-UP.

Note: * present the reaction point of vinyl double bonds.

The intension strength of PGA-UP after self-cured was $13.5 \mathrm{Mpa}$, close to the $15.6 \mathrm{MPa}$ of the one of an usual unsaturated polyester S-UP, which was synthesized with propanediol, m-phthalic acid and maleic anhydride as the raw materials(molar ratio as 2.2:1:1) in our lab [7]. The potential application of PGA-UP is inspiring, especially in the research field of medical polymer and tissue engineering, because of its self-curable properties.

\section{Conclusions}

One kind of novel unsaturated polyester PGA-UP with vinyl double bonds on the end of molecule chain is synthesized with GL and AC as the raw materials under very low reaction temperature. The PGA-UP product is a liquid mixture produced by different esterifying reaction degree of GL with AC. It demonstrated that the unsaturated polyester resin PGA-UP with $1 \%$ BPO could be self-cured when heated above $80^{\circ} \mathrm{C}$ without additional 
cross-linking monomers. It was speculated that the self cross-linking process carried on through the radical homopolymerization of the vinyl double bonds. The resin after self-cured presents good intension strength close to the usual unsaturated polyesters and its potential application is inspiring. This research also contributes to improve air pollution caused by volatile of cross-linking monomers.

\section{Acknowledgement}

Of China for the Youth (Grant No.51303164) and Shandong Province Outstanding Young and MiddleAged Scientists Research Awards Funds (Grant No.BS2011CL039).This work is sponsored by Natural Science Foundation

\section{References}

1. K. Q. Shen: Unsaturated Polyester Resin and its Application (Chemical Industry Press, Beijing, China, 2005)

2. N. Bicak, B. Karagoz, U. Tunca, J. Polym. Sci. Polym. Chem. Vol. 41 (2003),p.2549

3. N. Alemdar, B. Karagoz, A.T. Erciyes, N. Bicak*, Prog. Org. Coat. Vol. 60 (2007), p.69

4. S.Wang, M.J.Yaszemski, J.A.Gruetzmacher, L.Lu*,Polymer Vol. 49 (2008),p.5692

5. L.Cai, S. Wang, Polym. Vol. 51 (2010), p. 164.

6. N. Alemdar, B. Karagoz, A.T. Erciyes, N. Bicak*,J. Appl. Polym.Sci. Vol. 116 (2010),p.165

7. J.H. Meng, H.Y. Li *, Y.Y. Chen, Adv. Mat. Res. Vol. 699 (2013), p. 161. 